By the method of Sunyer i Balaguer [see Theorem A of the preceding paper] Theorem $\mathrm{B}$ can be improved and we can prove:

TheOREM C. Under the conditions of Theorem $\mathrm{B}, \tau_{S}=\tau$.

The following printing mistake in [1] may be noted:

In line 21 on page $215 e^{\rho\left(\sigma_{j}-\epsilon^{\prime}-\epsilon\right)}$ may be corrected to read $e^{\rho\left(k_{j}-\epsilon^{\prime}-\epsilon\right)}$.

\title{
REFERENCE
}

1. Q. I. Rahman, On entire functions defined by a Dirichlet series, Proc. Amer. Math. Soc. vol. 10 (1959) pp. 213-215.

NORTHWESTERN UNIVERSITY

\section{THE UNIVERSAL REPRESENTATION KERNEL OF A LIE GROUP}

\section{G. HOCHSCHILD}

Let $G$ be a connected real Lie group. The universal representation kernel, $K_{G}$, of $G$ is defined as the intersection of all kernels of continuous finite dimensional representations of $G$. Evidently, $K_{G}$ is a closed normal subgroup of $G$, and it is known from a theorem due to Goto (cf. [1, Theorem 7.1]) that $G / K_{G}$ has a faithful continuous finite dimensional representation. Thus $K_{G}$ is the smallest normal closed subgroup $P$ of $G$ such that $G / P$ is isomorphic with a real analytic subgroup of a full linear group. The known criteria for the existence of a faithful representation lead to a determination of $K_{G}$ which we wish to record here.

Suppose first that $G$ is semisimple. Let $\&$ denote the Lie algebra of $G$. Let $C$ stand for the field of the complex numbers, and denote by ${ }^{(5)}{ }^{c}$ the complexification of $(5)$, i.e., the semisimple Lie algebra over $C$ that is obtained by forming the tensor product, over the real field, of $\& 5$ with $C$. Denote by $S(\mathbb{S})$ and $S\left(\mathbb{S}^{C}\right)$ the simply connected Lie groups whose Lie algebras are $(S)$ and $B^{C}$, respectively. The injection $(B) \rightarrow B^{C}$ is the differential of a uniquely determined continuous homomorphism $\gamma$ of $S(\mathbb{B S})$ into $S\left(\mathbb{S}^{C}\right)$. The kernel $P$ of $\gamma$ is a discrete central subgroup of $S(\mathbb{S})$. Let $\phi$ denote the covering epimorphism of $S(\mathbb{S})$ onto $G$. We claim that $K_{G}=\phi(P)$, i.e., the universal representation kernel of the semisimple connected Lie group $G$ is the image, under the universal covering epimorphism, of the kernel of the canonical homomorphism $S(\mathbb{S}) \rightarrow S\left(\mathbb{S}^{C}\right)$.

Received by the editors September 18, 1959. 
Let $\rho$ be a continuous representation of $G$ in a full complex linear group $L$. The differential of $\rho$ is a representation of $(\$)$ which extends canonically to a representation of the complex Lie algebra $\left(S^{c}\right.$. This representation of $B^{C} C$ is the differential of a complex analytic representation $\rho^{*}$ of $S\left(B^{C}\right)$ (which is now regarded as a complex analytic group) in $L$ such that $\rho^{*} \circ \gamma=\rho \circ \phi$. Hence it is clear that $\phi(P)$ is contained in the kernel of $\rho$, and we conclude that $\phi(P) \subset K_{G}$.

On the other hand, let $F$ be the kernel of $\phi$, i.e., the fundamental group of $G$. Since $F$ is in the center of $S(\mathbb{S})$, it follows (by considering the adjoint representation of $S\left(B^{C} C\right)$ ) that $\gamma(F)$ lies in the center of $S\left(B^{C}\right)$. Since $S\left(B^{C}\right)$ is a semisimple complex analytic group, its center is finite, and there exists a complex analytic representation $\sigma$ of $S\left(B^{C}\right)$ whose kernel is precisely $\gamma(F)$ (cf. [3, 22-13, Theorem 4]). Now $\sigma \circ \gamma$ is a continuous finite dimensional representation of $S(\$)$ whose kernel is precisely $P F$. Hence $\sigma \circ \gamma$ yields a continuous finite dimensional representation of $G$ whose kernel is precisely $\phi(P)$. It follows that $\phi(P)=K_{G}$.

Now let $G$ be an arbitrary connected Lie group, and let $S$ be a maximal semisimple analytic subgroup of $G$. Let $A$ denote the closure in $G$ of $K_{S}$. By considering the adjoint representation of $G$, we see immediately that $A$ is contained in the center of $G$. Also, it is clear that $A \subset K_{G}$. Thus $K_{G}$ can be found from $G / A ; K_{G} / A=K_{G / A}$. Now $(S A) / A$ is a maximal semisimple analytic subgroup of $G / A$. Moreover, $(S A) / A$ isomorphic with the factor group of $S / K_{S}$ modulo the central subgroup $(S \cap A) / K_{S}$. Since $S / K_{S}$ is a semisimple connected Lie group with a faithful representation, its center is finite, and it follows that $(S A) / A$ has a faithful representation (cf. the two remarks on p. 934 of [2]). Hence, replacing $G$ with $G / A$, we may now assume that the maximal semisimple analytic subgroup $S$ of $G$ has a faithful representation.

With this assumption in force, let $G^{\prime}$ denote the commutator subgroup of $G$, and let $T$ be the closure in $G$ of the radical of $G^{\prime}$. Since every representation of $G$ is unipotent on the radical of $G^{\prime}$, we see at once that every compact subgroup of $T$ must lie in $K_{G}$, and thus also in the center of $G$. It follows that $T$ has a unique maximum compact subgroup $Q$ which is contained in $K_{G}$. We claim that $Q=K_{G}$. In order to see this, it suffices to show that $G / Q$ has a faithful representation. Let $U$ denote the radical of $(G / Q)^{\prime}$. Since $(G / Q)^{\prime}=\left(G^{\prime} Q\right) / Q$, it is clear that $U \subset T / Q$. Now $T / Q$ is a nilpotent connected Lie group that has no nontrivial compact subgroup. Hence $T / Q$ is simply connected. Hence every normal analytic subgroup of $T / Q$ is closed and simply connected. Thus $U$ is closed in $T / Q$, and hence in $G / Q$, and 
simply connected. On the other hand, the canonical image of $S$ in $G / Q$ is a maximal semisimple analytic subgroup of $G / Q$ and has a faithful representation, because $S$ has a faithful representation (the argument being the same as the one used before). Now it follows from a result due to Goto (cf. [2, Theorem 4.2]) that $G / Q$ has a faithful representation, and we conclude that $K_{G}=Q$.

Our result is the following: let $G$ be a connected Lie group, and let $S$ be a maximal semisimple analytic subgroup of $G$. Let $A$ be the closure in $G$ of $K_{S}$. Then $K_{G}$ is the subgroup $P$ of $G$ that contains $A$ and is such that $P / A$ is the unique maximum compact subgroup of the closure in $G / A$ of the radical of $(G / A)^{\prime}$.

The corresponding problem for complex analytic representations of complex analytic groups appears to be rather different. If the complex analytic group $G$ has a simply connected radical then $G$ has a faithful complex analytic representation, as we shall see below. The other extreme is illustrated most simply with the compact complex analytic groups, which have no nontrivial complex analytic representations. However, even in the abelian case, a complex analytic group may fail to have nontrivial complex analytic representations for much less transparent reasons. Perhaps the difficulty is illustrated best by the following example (which carries out a suggestion made to me by M. Rosenlicht):

Let us consider the additive group $(C, C)$ of a two dimensional complex vector space. Let $L$ denote the subgroup of $(C, C)$ that is generated by the three elements $(1,0),(0,1)$, and $(\alpha i, i)$, where $\alpha$ is real and irrational. Let $G$ be the factor group $(C, C) / L$, regarded as a complex analytic group in the natural fashion. We shall show that $G$ has no nontrivial complex analytic representation. Let $\rho$ be any complex analytic representation of $G$. If $Z$ denotes the additive group of the integers then $G$ is the factor group of the direct product of two copies of $C / Z$ modulo the subgroup generated by the element $\left((\alpha i)^{\prime}, i^{\prime}\right)$, where the prime indicates "coset $\bmod Z$." Since $(C / Z, C / Z)$ has a compact real form, it follows that $\rho$ must be semisimple. Since $G$ is commutative, $\rho$ is therefore a diagonal representation. Hence, in order to show that every complex analytic representation of $G$ is trivial, it suffices to show that every complex analytic homomorphism of $G$ into the multiplicative group $C^{*}$ of the nonzero complex numbers is trivial. In other words, it suffices to show that every complex analytic homomorphism $\phi$ of $(C, C)$ into $C^{*}$ whose kernel contains $L$ is trivial. Now we have $\phi(x, y)=\exp (u x+v y)$, for all $(x, y) \in(C, C)$, where $u$ and $v$ are fixed complex numbers. Since $\phi(1,0)=1=\phi(0,1)$, we must have $u=2 \pi p i$ and $v=2 \pi q i$, where $p$ and $q$ are integers. Since 
$\phi(\alpha i, i)=1$, we must have $p \alpha+q=0$, and by our assumption on $\alpha$ this gives $p=0$ and $q=0$, so that $\phi$ is trivial. Thus we have shown that $G$ has no nontrivial complex analytic representation.

On the other hand, $G$ has no compact complex analytic subgroup other than (1). In order to see this, it suffices to show that no one dimensional complex subspace of $(C, C)$ contains two elements of $L$ that are linearly independent over the real field. Let $r, s, t$ be integers not all 0 , and consider the $C$-subspace of $(C, C)$ that is spanned by the element $r(1,0)+s(0,1)+t(\alpha i, i)=(r+t \alpha i, s+t i)$ of $L$. Let $a$ and $b$ be real numbers. Then the product by $a+b i$ of our element of $L$ is $(a r-b t \alpha+(a t \alpha+b r) i, a s-b t+(a t+b s) i)$. If this is an element of $L$ there are integers $r_{1}, s_{1}, t_{1}$ such that

$$
\begin{aligned}
a r-b t \alpha & =r_{1}, & a t \alpha+b r & =t_{1} \alpha, \\
a s-b t & =s_{1}, & a t+b s & =t_{1} .
\end{aligned}
$$

From the second and the fourth of these we obtain $b(r-s \alpha)=0$. If $b=0$ then our second element of $L$ is a real multiple of the element of $L$ that we started with. If $b \neq 0$ then $r-s \alpha=0$, and our assumption on $\alpha$ gives $r=0$ and $s=0$. The above relations now reduce to $r_{1}=-b t \alpha$, $s_{1}=-b t, t_{1}=a t$. The first two of these give $r_{1}=s_{1} \alpha$, whence $r_{1}=0$ and $s_{1}=0$. Since $t_{1}=a t$, our second element of $L$ is again a real multiple of the element we started with. Thus we conclude that $G$ has no nontrivial compact complex analytic subgroup.

The above difficulty is avoided if we assume that the complex analytic group $G$ coincides with its commutator subgroup $G^{\prime}$. Now let $K_{G}$ denote the intersection of all kernels of complex analytic representations of $G$. We shall prove that, if $G=G^{\prime}$, then $K_{G}$ coincides with the smallest complex analytic subgroup of $G$ containing the unique maximum compact subgroup of the radical of $G$.

Let $R$ denote the radical of $G$. Since $G=G^{\prime}, R$ is nilpotent, so that $R$ has a unique maximum compact subgroup $Q$. Let $Q^{*}$ be the smallest complex analytic subgroup of $R$ that contains $Q$. Since every complex analytic representation of $G$ is unipotent on $R$, it is clear that $Q^{*} \subset K_{G}$. Let us regard $R$ as a real connected Lie group, for a moment. $Q$ is in the center of $R$, and $R / Q$ is simply connected, because it contains no nontrivial compact subgroup. $Q^{*} / Q$ is a real analytic subgroup of $R / Q$ and lies in the center of $R / Q$. Since $R / Q$ is simply connected, $Q^{*} / Q$ is closed in $R / Q$, and the factor group is simply connected. It follows that $Q^{*}$ is closed in $R$, and hence also in $G$, and $R / Q^{*}$ is simply connected. Now $R / Q^{*}$ is the radical of $G / Q^{*}$, and there remains to prove that $G / Q^{*}$ has a faithful complex analytic representa- 
tion. Hence it suffices to prove that if the radical $R$ of a complex analytic group $G$ is simply connected then $G$ has a faithful complex analytic representation.

In order to prove this, let $S$ be a maximal semisimple complex analytic subgroup of $G$. Since the center of $S$ is finite, so is $S \cap R$. It follows that $G$ is complex analytically isomorphic with a factor group of the appropriately defined semidirect product $S \cdot R$ modulo a finite subgroup. Hence (cf. [2, p. 934, Remark 2]) it suffices to show that the semidirect product $S \cdot R$ has a faithful complex analytic representation. Since $R$ is simply connected, there is a faithful complex analytic representation of $R$ that is unipotent on the maximum nilpotent normal analytic subgroup of $R$, and, a fortiori, on the subgroup generated by the commutators $y x y^{-1} x^{-1}$, with $y \in G$ and $x \in R$. For a proof in the real case, see [2, Theorem 4.1]; the complex case can be handled in precisely the same way. Moreover, the extension theorem for representations of real analytic groups, [2, Theorem 3.1], holds also, with the same proof, for complex analytic representations of complex analytic groups. Hence our representation of $R$ can be extended to a complex analytic representation of $G$ that is faithful on $R$. If we take the direct sum of this representation with a complex analytic representation of $S \cdot R$ whose kernel is $R$, which exists because $S$ has a faithful complex analytic representation, we obtain a faithful complex analytic representation of $G$. This completes the proof of our above assertions.

\section{REFERENCES}

1. G. Hochschild and G. D. Mostow, Representations and representative functions of Lie groups, Ann. of Math. vol. 66 (1957) pp. 495-542.

2. - Extensions of representations of Lie groups and Lie algebras, I, Amer. J. Math. vol. 79 (1957) pp. 924-942.

3. —_ Séminaire "Sophus Lie," École Normale Supérieure, Paris, 1954-1955.

University of California, Berkeley 